pleted, he will enter upon his everlasting rest, re. spected, loved and honored by his contemporaries, while his memory will be cherished by a grateful posterity.

\section{TWO CASES OF TRISMUS NASCENTIUM SUCCESSFULLY TREATED BY TETANUS ANTITOXIN.}

BY JACOB FRIEDMAN, M.D.

PROFESSOR OF DISEASES OF CHILDREN AND CLINICAL MEDICINE, BEAUMONT MEDICAL COLLEGE.

$$
\text { ST. LOUIs, Mo. }
$$

Within the past five weeks I have had an opportunity of experimenting with tetanus antitoxin in the treatment of two cases of trismus nascentium. This disease of infancy has been almost uniformly fatal and, notwithstanding the fact that all kinds of treatment have been used, has thus far resisted every measure.

On learning of the successful treatment of tetanus in the adult by the serum treatment, I determined to try the same remedy for the infantile disease. By reason of the extreme youth of the patient one has no guide as to the quantity of the serum to be used. As will be seen in the first case, knowing that the child would die under the ordinary plan of treatment, a large dose was used in the beginning, considering the weight of the child as compared with that of the adult, in which a full bottle is usually administered.

In both instances the serum used was of Gibier's make, the product of the New York Biological Institute. The manufacturers give directions to the effect that the entire contents of one bottle should be used every six hours until improvement sets in. In both cases two bottles were used. and all the serum was absorbed within six hours. There were no rise of temperature, no abscess and no eruption of any character Immediate improvement resulted after each injection, and this was made more noticeable by the fact that the children were at the worst in the morning. The convulsions therefore appeared in the morning, since the children had received no injection during the night. During the day both children rested well. Their convulsive movements, or convulsions, were the first symptoms that yielded. In addition to the serum, both children were given from $\frac{1}{2}$ to 1 grain of chloral every three hours.

Case 1.-Girl ten days old, parents poor, and consequently their surroundings were not of the cleanest. Umbilicus slightly suppurating, jaws thoroughly locked, inability to nurse, fever, convulsions, etc. On seeing case, one-third of a bottle of serum was immediately used, and this was repeated in six hours. As the last injection was given at $6: 30$ P.M., the child had no serum until 9 o'clock the next morning, when a further injection was given, and as the improvement seemed marked, only one-sixth of a bottle was given at the next injec. tion. As before stated, two bottles were used. Patient has entirely recovered.

Case 2.-Boy fourteen days old, conditions and symptoms similar to first case. I proceeded exactly as in the first case and obtained the same results. Both patients are nursing again, and were able to begin nursing after three days treatment.

I have during my practice seen a large number of these cases, which have come both from dispensary practice and a large charity practice among the poor Russian emigrants which I did in my early professional life. Heretofore, I have seen only one case recover. The successful issue of the two cases reported is very gratifying, and certainly will lead me to adopt the same method in the treatment of similar cases.

2804 Clark A venue.
THE OPHTHALMOSCOPE AS AN AID IN GENERAL MEDICAL DIAGNOSIS.

Read before the Indiaua State Medical Society, May, 1897. BY L. D. BROSE, M.D., Ph,D.

OCUIIST AND AURIST ST, MARY'S HOSPITAL, EVANSVILLE, IND.

Every man, in the general practice of medicine, is continually meeting with patients whose symptoms are obscure and the question of diagnosis is long unsolved. A careful ophthalmoscopic examination in these cases may at once detect hidden eye lesions that not only serve to identify the general disorder but also furnish us a correct prognosis. Perhaps the relation of a few cases at the outset will make the subject more interesting and impress you with the importance of giving this matter your close attention.

Joseph G., aged 27 years was referred to me by Dr. Edwin Walker. He complains of seeing very poorly, and of headache in the back and sides of the head. The left leg is often numb and there is a pricking sensation to the feet as if his shoes contained pins. The patella reflex is present and the urine negative. The pupils react to light, while the general external eye appearances are normal. With the ophthalmoscope both optic discs are clearly defined but of pale color, indicative of primary atrophy. The blood vessels are normal in size and without discoverable pathologic lesion. Field of vision is contracted peripherally and there is a central scotoma in either oye. He counts fingers at five feet. Diagnosis: Multiple sclerosis.

H. G., a German 38 years old and married, comes with a history of double vision during the past twelve months. He admits having had syphilis nineteen years ago. Patella reflex present. Both pupils strongly contracted and without reaction to light. The left external rectus muscle is paralyzed and both optic nerves are atrophied. With the right eye he sees 15 cc and with the left eye $15 \mathrm{xx}$. The field of vision shows peripheral contraction with islets of color scotoma for green and red. Diagnosis : Locomotor ataxia.

Mr. L. H., unmarried, 21 years old, was sent to me by Dr. H. T. Dixon, May 23, 1891, because of poor eyesight. $\mathbf{H e}$ had been at work up to within a few days and was not aware he had any serious trouble other than that of sight. Vision in the right eye was reduced to recegnizing hand movements, and in the left eye to counting fingers at eight feet. Ophthalmoscopic examination detected retinitis in both eyes with radiating whitish patches around the macular regions and numerous retinal hemorrhages. Both optic discs were swollen and their outlines obliterated. The urine was highly albuminous with many granular tube casts. Diagnosis : Bright's disease of the kidney. Patient was unable to come to my office again but was attended by his regular physician, death ensuing within three weeks from the time he first consulted me

F. K., a farmer, was sent to me Aug. 24, 1895, with the history that several months before his eyesight became so bad that he could scarcely read. The vision then slowly improved again but now had relapsed so that patient was advised to consult an oculist. With the right eye he counted fingers at ten feet and with the left eye he saw 15-c. The ophthalmoscope showed both optic nerves swollen, very indistinctly outlined, many small retinal hemorrhages and whitish star-shaped patches around the central zones of vision. The urine was highly albuminous and with a specific gravity of 1010 . His physician was advised that the patient had albuminuric retinitis and that in all probability he would die within twelve months. He did not accept our prognosis, but informed the man, who looked in the best of health, that he would treat his case himself and that he might expect to recover his sight. In less than six months the patient died.

C. B., 44 years old, came to see me the first time in 1893 , saying he had trouble with his eyes. His general health was not good although he continued attending to his law practice. $\mathrm{He}$ has steadily lost flesh during the past two years, notwith. standing his appetite was good. Vision in the right eye $15 \cdot \mathrm{xv}$, in the left eye $15 \mathrm{xl}$. He was slightly hyperopic and with the correcting lens for this and his presbyopia read Snellen 11 -11 both eyes. The ophthalmoscopic examination disclosed in both retina, retinitis with whitish patches in the region between the disc and macula. No hemorrhages were discovered, and the outlines of the optic nerves were clearly defined. The urine showed a trace of sugar. Diagnosis : Retinitis diabetica. In 1894 he made a visit to Hot Springs and returned with his vision greatly improved, the whitish patches however had not disappeared. Patient died a year later. 
H. H. B., 40 years old, consulted me Jan. 12,1892 , because of disturbed vision in the left eye. With the right eye he saw $15 \cdot \mathrm{xxx}$ and with +2.5 correcting his hyperopia he saw $15 \cdot \mathrm{xv}$. Sight in the left eye was reduced to counting fingers at twelve feet. The ophthalmoscope revealed retinal hemorrhages in both eyes with disease of the retinal blood vessels. Patient denied ever having had syphilis, nor has he ever been addicted to the use of alcohol. The urine was free from sugar and albumin. Diagnosis: Retinitis hemorrhagica, due to disease of the retinal blood vessels. Patient was cautioned to give up much of his business and take a prolonged vacation in the early future. Internally, iodid of potash was prescribed. The advice however was not heeded and the vision in the left eye rose with $+2,5$ lens to $15-\mathrm{lxx}$. Within a year he had a slight cerebral hemorrhage, followed in another twelve months by a second that terminated his existence.

In our ophthalmoscopic examinations of the optic nerve we recognize two kinds of atrophy. Such as have been occasioned without inflammatory neuritis and hence termed primary or genuine atrophy; and such as have been preceded by swelling of the nerve fibers and other evidences of inflammation and termed consecutive atrophy. The causes of primary atrophy of the optic nerve are to be sought for in disease of the brain or spinal cord, or in disease located in the course of the optic nerve itself, which through injury or pressure has its conducting function interfered with. In general paralysis of the insane, primary atrophy of the optic nerve may occur at a very early period and its significance is then of great diagnostic and prognostic importance. The general nerve appearances are very like those seen in locomotor ataxia. One difference though may at times be found in a slight preliminary cloudiness of the papilla and adjacent retina. ${ }^{1}$ Gowers says he has observed this a few times but that much oftener it has been wanting. Reflex rigidity of the pupil may long continue as a prodromal symptom of the disorder. Other eye muscles may very early and in seeming perfect health be paralyzed singly or in groups, which paralysis is often temporary but subject to relapses. The visional field, unlike that in tabes, is apt to show concentric narrowing, with central interference in color conduction. Eye symptoms in multiple sclerosis are often quite characteristic and of the greatest value in establishing a diagnosis. Nystagmus and tremor when fixing an object must be looked for and are occasioned through insufficient innervation of the nuclei of the ocular muscles. Disturbance is found in the peripheral visional field in an irregular narrowing and may be concentric, with central color scotoma that seldom becomes absolute. ${ }^{2}$ The impairment of sight may appear at the beginning of the disease and may even remain the sole manifestation of the disorder for a long time. The characteristic is frequent improvement and relapse in keeping with improvement or a growing worse of the patient's general condition. The scotoma may be one-sided, again peripheral, then disappear and reoccur central. The optic disc is changed to a grayish white color, and later becomes excavated so that the lamina cribosa is seen. The disc outlines however remain clearly defined while the blood vessels of the fundus are in general little altered in caliber or course.

The frequency of optic nerve atrophy in locomotor ataxia is variously estimated. Gowers mentions 13 per cent.; Galezowski says about two-thirds of all primary optic nerve atrophies are due to tabes, while Charcot places it still higher and says that in nearly every case, even if spinal symptoms are at first absent,

1 Medical Ophthalmoscopy.

2 Knies: The Eye in General Diseases. they will appear at a future period. Prof. Uhlhoff, now of Breslau, who perhaps has done more original investigating in this line than any other oculist. says not more than one-half of the cases of primary atrophy are associated with disease of the spinal cord. Ophthalmoscopically we see a gray discoloration of the optic papilla beginning, as a rule, in the outer half; it extends slowly over the entire nerve, which later becomes excavated, so that the meshes of the lamina cribrosa may be seen. The field of vision shows peripheral contraction perhaps in islets or sectors but we especially look for contraction in the outer and upper part of the field. Central positive scotoma hardly ever occur and should make us doubtful of our diagnosis. Disturbance in the color field is important and has a bearing on the prognosis. Where the color narrowing is relatively very much greater than for white, the the disease may be expected to progress rapidly. Conversely, contraction of the color boundaries in keeping with the rest of the field denotes a slowly progressive or stationary disease. There is great disproportion between the visible atrophy of the optic dise and the disturbance of vision. ${ }^{2}$ As a rule, however, when the discoloration of the disc is much greater than we might expect from the amount of disturbance of vision, the case is apt to be slowly progressing. The ophthalmoscope may reveal total gray discoloration of the disc although there is hardly any disturbance of vision. Muscular disorders of all kinds occur with great frequency in tabes, and are nuclear or peripheral in nature. In fact every paralysis of an eye muscle, occurring suddenly in a person in health and without an injury, should arouse a suspicion of a beginning tabes, the more so if it recovers in a comparatively short time or subsequently relapses. According to Kahler of Vienna, abducens palsy is the most frequent, ptosis next. Pupillary symptoms are very frequent and of great diagnostic value. Absence of light reaction, followed by loss of reaction to accommodation and convergence, is one of the most characteristic signs of beginning posterior spinal sclerosis. Consecutive atrophy of the optic nerve is the final stage of a papillitis or retinitis and is due to the newly formed connective tissue in and between the nerve fibers, interfering with their nutrition. During the active stage of the disease the nerve head is swollen, its margins obliterated or very indistinct, and the color reddish, approaching that of the fundus. Through mechanical congestion the veins become distended and tortuous, while the arteries are either unchanged or smaller. As the inflammatory symptoms decline the outlines of the disc are again seen, and may grow irregular or even smaller than in health. The color changes to white but the lamina cribrosa remains invisible. The blood vessels now grow smaller and many are enclosed by whitish lines. Intracranial disease, such as tumors, menigitis, brain abscess and cerebral softening from vascular obstruction, is by far the most common cause of consecutive atrophy of the optic nerve. Systemic disease associated with the retention of morbific or toxic material in the blood may likewise occasion optic neuritis. Local lesion in the orbit may cause a unilateral form. Retinitis is in most cases a symptom, the proper interpretation of which will lead to the detection of its cause in some constitutional disorder. To such causative disorders we first place albuminuria, then diabetes, syphilis, blood diseases like lencocythemia and disease of the blood vessels. Retinitis albuminurica is characterized, 
in addition to the common symptoms of retinal inflammation, such as diffuse retinal cloudiness, veiling of the disc outlines and blood vessels with frequent congestion and tortuosity of the same, by white spots or patches of fatty degeneration of the retinal elements and cellular exudate. These white spots are found characteristic in two places, in circles around the optic nerve, or radiating star.like around the macula. Especially typical is the latter stellar-like arrangement of the white spots. Hemorrhages may be found in all parts of the retina, coming chiefly from the retinal veins and capillaries, as shown by the striated appearance of the extravasated blood. Every form of nephri. tis may give rise to retinal trouble; however it is found oftenest with the cirrhotic or interstitial kidney disease. The relation between the eye lesion and the kidney disease is that the retention of waste matter in the blood sets up a disease of the blood vessels of the eye and as a sequence inflammation and degeneration of the retina. The intensity of the eye lesion bears no fixed relation to the severity of the nephritis nor to the amount of albumin in the urine. On the contrary the eye lesion may improve while the kidney disease is growing worse or the kidney disease improve and the eye lesion grow worse. Nevertheless, retinitis renders the prognosis very grave in albuminuria, and it is a fact that most pationts die within one year after the eye lesion has been recognized by the ophthalmoscope. Uremic transitory blindness is a different trouble than the one we have just considered and is accompanied by vomiting, convulsions and the general symptoms usually present in uremic poisoning. Retinitis diabetica is, following Hirschberg, ${ }^{3}$ best depicted under two principal types. Where we find whitish spots in the retina lying between the upper and lower temporal branches of the central artery and to the temporal and nasal side of the optic nerve. As the patches enlarge they become striated or crescent shaped. The star like patches as seen in albuminuric retinitis are never present without the urine contains albumin as well as sugar. Likewise in and around the white spots do we note the absence of pigment degeneration which is so common in syphilitic retinal trouble. Small hemorrhagic points, lines or spots may be found in all parts of the retina and even a hemorrhagic spot may rest upon one of the whitish exudates. The fact that the hemorrhages do not overstep a certain degree of severity is characteristic. The hemorrhages may undergo reabsorption, but Hirschberg says he has never seen the whitish patches wholly disappear, notwithstanding improvement in vision and the general symptoms. The absence of diffuse retinal cloudiness and the non-involvement of the optic disc with veiling of its outlines, are of great value in differentiating this trouble from albuminuric retinitis. ${ }^{2}$ The second type of the disease is the purely hemorrhagic, spontaneous hemorrhages occur and are due to sclerosis of the blood vessels, not only in the retina but in the brain and other parts of the body, Subconjunctival hemorrhage in an apparently healthy person may have a connection with diabetes; likewise a chronic and persistent inflammation of the eyelids. Hemorrhage into the vitreous may happen with or without hemorrhagic glaucoma diabetic in origin. Cataract is a frequent accompaniment of diabetes in both the young and the old. In addition, diabetics have trouble in accommodation and require frequent changing of their reading lenses and a strength out of

\footnotetext{
${ }^{3}$ Deutsche medicinische Wochenschrift, No. $51,1890$.
}

all proportion to their presbyopia. A very rapidly developing myopia without lens opacity is sometimes found in persons long past middle life. While our purely hemorrhagic type is less characteristic of diabetes than is our exudative, its prognostic value, so far as the preservation of sight and even life is concerned, is more grave. Hemorrhagic retinitis independent of diabetes and albuminuria or alteration of the qualtity of the blood, but associated with vascular derangement such as general arterial sclerosis or endarteritis need interest us here only for the unfavor. able prognostic value it has upon the patient's life expectancy.

\section{REMARKS ON OTITIS MEDIA AND OTITIC SYPHILIS, WITH REPORT OF A RHIN. OLITH AND AN ACCESSORY TOOTH IN THE NOSE.}

Read before the South Carolina Medical Association at Union, S. C., A pril 26, 1897

BY EDWARD F. PARKER, M.D.

PROFESSOR OF PHYSIOLOGY AND ASSYSTANT IN DISEASES OF THE EYE, EAR, NOSE AND THROAT, MEDICAL COLLEGE OF SOUTH CAROLINA. cF A RLESTON, s. c.

The etiology, symptoms and pathologic changes of an ordinary case of acute suppurative inflammation of the middle ear are more or less familiar to us all. The formation of pus in the tympanic cavity is usually followed by rupture of the drumbead and the discharge heralds an approaching sure and speedy recovery if drainage is free enough to permit the early exit of the pus. Silch a case, free from complications, pursues a regular course and tends under careful hygienic and rational treatment, to spontaneous cure. Often, however, we meet with cases when for some reason the tympanic membrane does not yield to the pressure and the pus after invading the mastoid antrum and cells, may have to seek another outlet. The Eustachian tube, the natural ventilator of the middle ear, rarely serves as a drainage tube on account of the closure of its small lumen from inflammatory swelling, originating usually in the naso-pharynx. In such cases the bony tissues are necrosed and the pus seeks an outlet in the cranial cavity or some of the venous sinuses or arteries passing in close proximity to the attic. Again, after rupture of the drumhead the opening may not be large enough to permit free drainage and the tympanic mucous membrane being constantly bathed in a septic purulent fluid becomes subject to polypoid degeneration; and for this or other reasons the discharge becomes chronjc. It is to two of these complications that I invite your attention with illustrative cases.

W. E. N., aged 24, of Dock, S. C., consulted me by advice of Dr. Steele, on March 11, 1897, for deafness in and a chronic discharge from the right ear. Two months previously he suffered from an attack of grippe with violent pains in both ears. The right began to discharge ten days after and had not stopped since. In the interval he had recurrent attacks of severe mastoiditis. On examination the skin over the mastoid was found swollen, boggy and painful on pressure. Hearing was reduced to two thirty-six ths in the right ear and the external canal was closed by a soft fluctuating tumor with a fistulous tract opening on its surface. The drumhead, not at first observable, was subsequently found intact. The fistula communicated with the mastoid cells and the bony canal was considerably necrosed. A free incision was made through the tissues of the external auditory canal and the tract and adjacent diseased bone thoroughly curetted and packed with iodoform gauze. Free drainage was thus established and three weeks after, the patient reported his hearing and his ear normal in every respect. 